\title{
Band Structure of Mn/ZnTe Studied by Angle-Resolved Photoelectron Spectroscopy
}

\author{
B.J. Kowalski ${ }^{a}$, B.A. Orlowski ${ }^{a}$, M. PietrzyK $^{a}$, \\ P. $\mathrm{KACZOR}^{a}$, K. Kopalko ${ }^{a}$, S. Mickievicius ${ }^{b}$ \\ AND R.L. JOHNSON ${ }^{c}$ \\ ${ }^{a}$ Institute of Physics, Polish Academy of Sciences \\ al. Lotników 32/46, 02-668 Warsaw, Poland \\ ${ }^{b}$ Semiconductor Physics Institute, A. Gostauto 11, 2600 Vilnius, Lithuania \\ ${ }^{c}$ II Institute of Experimental Physics, University of Hamburg \\ Luruper Chaussee 149, 22761 Hamburg, Germany \\ The electronic band structure of $\mathrm{Mn} / \mathrm{ZnTe}(110)(1 \times 1)$ has been studied \\ by angle-resolved photoelectron spectroscopy. The sets of spectra were ac- \\ quired for the clean surface and after in situ deposition of $0.4 \mathrm{ML}$ of $\mathrm{Mn}$, in \\ order to compare the band structures and to reveal changes brought about by \\ the presence of $\mathrm{Mn}$. The experimental band structure diagram of $\mathrm{Mn} / \mathrm{ZnTe}$ \\ along the $\Gamma-K$ direction in the Brillouin zone has been derived from the \\ experimental data. Indications of interaction between the Mn $3 d$ states and \\ $s p^{3}$ bands of the semiconductor are discussed.
}

PACS numbers: 79.60.-i, 71.23.-k

\section{Introduction}

ZnMnTe attracts considerable interest in the context of search for ferromagnetic semiconductors suitable for spintronic devices. Theoretical considerations showed that free carrier-induced ferromagnetism may occur in II-VI compounds [1]. As a consequence, investigations of effective $p$-type doping of ZnMnTe are under progress [2]. Moreover, effective spin injection from ZnMnTe to nonmagnetic semiconductor was demonstrated [3]. The band structure of ZnMnTe, 
in particular distribution of $\mathrm{Mn} 3 d$ states, is an important piece of information for a detailed description of interaction between magnetic ions, crucial for magnetic properties of the material.

We report on the results of band structure study of the Mn/ZnTe system prepared in situ by deposition of submonolayer amounts of Mn on the $\mathrm{ZnTe}(110)$ surface under ultra high vacuum conditions. In accordance with data previously obtained for other II-VI compounds [4], deposited Mn atoms react with the surface of the substrate and a thin subsurface layer of Mn-doped crystal is formed. Therefore, we could study ZnTe and ZnMnTe for the same sample and under the same experimental conditions. So the comparison of the results was more reliable and enabled us to reveal the Mn $3 d$ contribution to the valence band structure.

\section{Experimental conditions}

The angle-resolved photoemission experiments were performed in the 15-30 eV photon energy range at the F2.2 beamline at HASYLAB, Hamburg (Germany). The radiation was linearly polarized, incident at $45^{\circ}$. The clean surface of a ZnTe wafer was prepared by $\mathrm{Ar}^{+}$ion bombardment and annealing under UHV conditions. The surface crystallinity was assessed by low-energy electron diffraction (LEED). 0.4 ML of Mn was deposited on the ZnTe surface from an effusion cell. The reported energy distribution curves were acquired for off-normal configuration. The band structure of the system was mapped along the (110) direction in the Brillouin zone of ZnTe.

\section{Results and discussion}

Figure 1 shows the sets of photoemission spectra measured for clean $\mathrm{ZnTe}(110)$ surface and for the same surface covered with 0.4 ML of Mn. The scattered electron background, determined by the Shirley method, has been subtracted. The origin of the energy scale was set at the Fermi level, as measured for a thick Mn layer. The binding energy range of the spectra covers the valence band of ZnTe. The spectral features related to the bands of the crystal can be followed for both clean and Mn-enriched surfaces. However, Mn deposition caused some blurring of the maxima. A shift of the spectra by $0.5 \mathrm{eV}$ can be attributed to a downward surface band bending caused by the Mn deposition. It is reasonable for $\mathrm{Mn}$ with relatively low work function $(4.1 \mathrm{eV}[5])$ deposited on $n$-type $\mathrm{ZnTe}$ $(\phi=5.43 \mathrm{eV}[6])$. An additional maximum appearing at about $3 \mathrm{eV}$ below the valence band maximum $\left(E_{\mathrm{VBM}}=1.7 \mathrm{eV}\right.$ for $\left.\mathrm{Mn} / \mathrm{ZnTe}\right)$ can be discerned in the spectra recorded for $\mathrm{Mn} / \mathrm{ZnTe}$ at the detection angle of $26-32^{\circ}$.

Figure 2 shows a comparison of the spectra taken for both surfaces at the angles of 0,12 , and $24^{\circ}$. An increase of emission in the whole valence band can be observed for all these angles. However, for $24^{\circ}$ a maximum increase of 

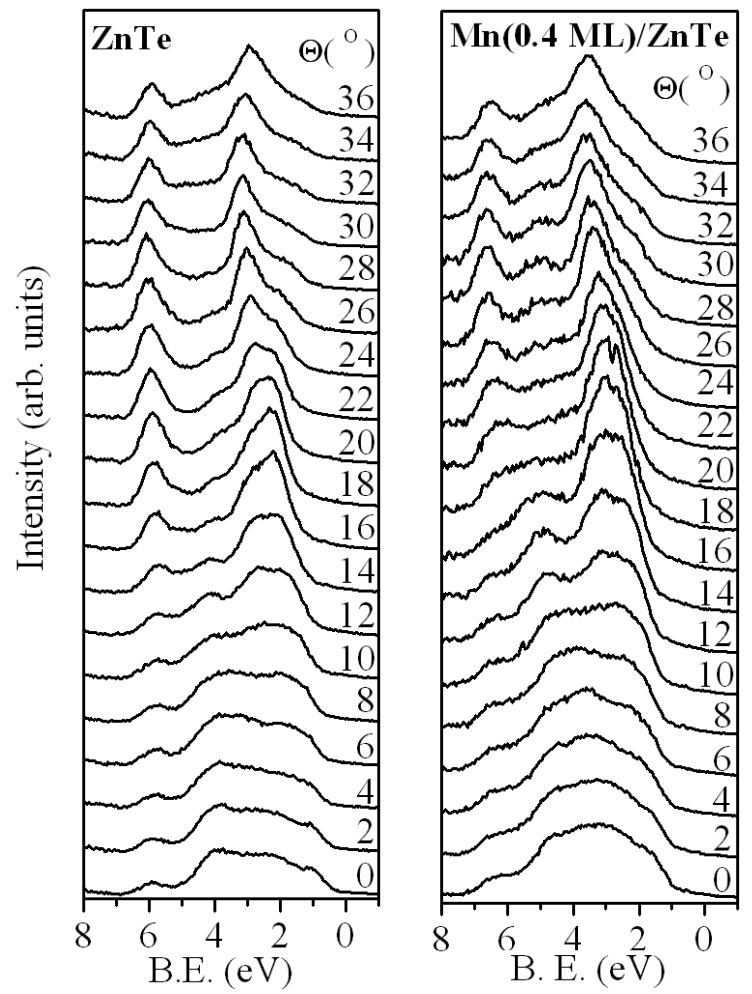

Fig. 1. Sets of angle-resolved photoemission spectra taken for $\mathrm{ZnTe}(110)$ surface clean (left) and after deposition of $0.4 \mathrm{ML}$ of Mn (right). The photon energy was $23 \mathrm{eV}$, the energy axis origin was set at the Fermi energy. The change of the detection angle corresponded to scanning the band structure along the $\langle 110\rangle$ direction in the Brillouin zone.

emission occurs in the top part of the valence band and at about $5 \mathrm{eV}$. These spectra correspond to emission from the states lying far away from the centre of the Brillouin zone.

The band structure and photoemission spectra of a zinc-blende diluted magnetic semiconductor were calculated by Mašek and Velický [7]. A parametrized tight-binding model and the coherent potential approximation were applied to describe spectral densities and some angle-resolved photoelectron spectra (AR PES) of $\mathrm{Cd}_{0.5} \mathrm{Mn}_{0.5} \mathrm{Te}$. The main feature revealed was the appearance of Mn $3 d$ resonance at about $3 \mathrm{eV}$ below the valence band edge. The $d$ states with $e_{\mathrm{g}}$ symmetry were only slightly broadened by hybridization, while $t_{2 \mathrm{~g}}$ states strongly hybridized with the Te $5 p$ states. They contributed to the density of states in the whole valence band, appearing considerably at the valence band maximum. However, the photoemission from Mn $3 d$ states became perceptible only for the photon energies higher than $40 \mathrm{eV}$ (due to the increase of the photoexcitation cross-section). 


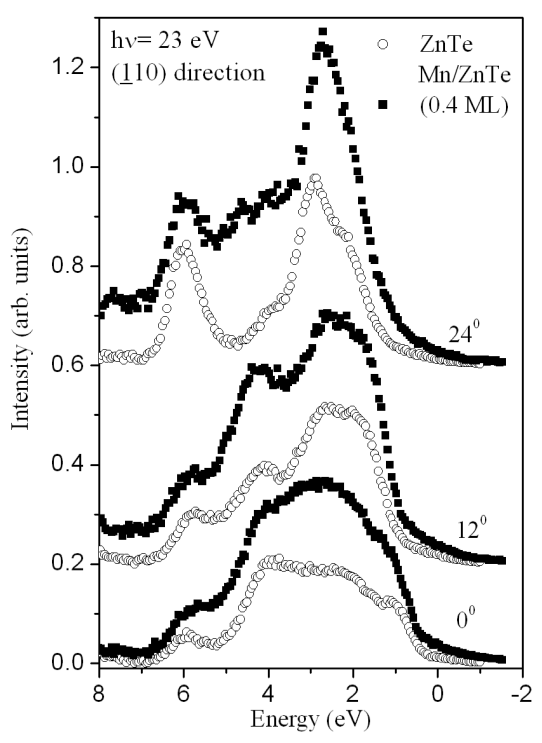

Fig. 2. A comparison of corresponding photoemission spectra obtained for clean and Mn-doped ZnTe(110) surface for the detection angle of 0 (normal emission), 12 and $24^{\circ}$.

Therefore, for lower photon energies, usually used for angle-resolved photoemission experiments, some indirect manifestations of Mn $3 d$ contribution to the band structure have to be analyzed. However, the most important consequence of the introduction of Mn into the crystal was distortion of the flat $d$ band and the lower branch of the valence band in the region where they overlap, e.g. in the middle of the $\Gamma-K$ line in the Brillouin zone. The non-crossing rule led to the appearance of a discontinuity in that band of the parent semiconductor.

The contribution of Mn $3 d$ states to the valence band can be discerned in Fig. 2. The maximum increase of emission noticeable for $24^{\circ}$ at the valence band maximum and at the binding energy which may correspond to the position of the weakly hybridized $d_{\mathrm{e}}$ states is consistent with expectations of Mašek and Velický [7].

In order to emphasize modifications of the band structure due to overlap of Mn $3 d$ states and $s p^{3}$ bands, the band structure diagram (Fig. 3) was derived from the set of off-normal photoemission spectra measured for the $\Gamma-K$ direction in the Brillouin zone. The curves, a Shirley background subtracted, were deconvoluted into sets of Gaussians. The binding energy positions of the Gaussians corresponding to features that could be revealed in the spectra were used to derive an experimental band structure. The corresponding values for $k$ vector were calculated within the free-electron final-state model. The overall shapes and relative energy positions of the bands represented by experimental points correspond reasonably well to those calculated by Walter et al. [8]. However, a noticeable deviation occurs in the deeper part of the band. A discontinuity at the binding 


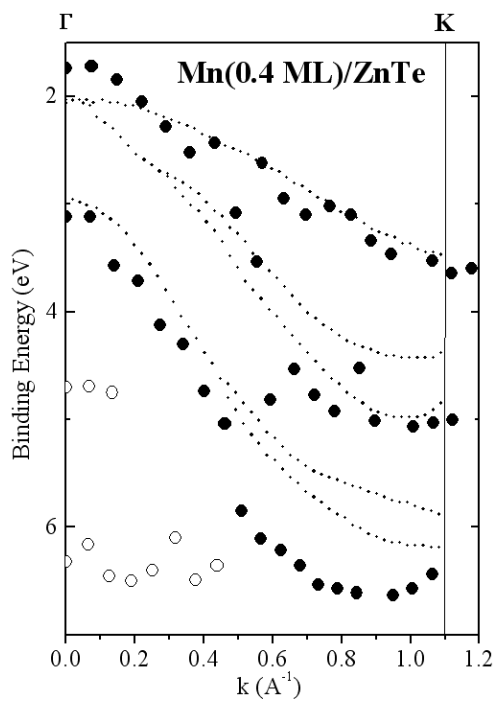

Fig. 3. The experimental band structure diagram along the $\langle\underline{1} 10\rangle$ direction in the Brillouin zone derived from the set of angle-resolved photoemission spectra obtained for Mn-doped ZnTe(110) surface (Fig. 1). The full dots correspond to features related to the bulk bands, open symbols mark other features, probably corresponding to density-of-states effects (band-edge-emission) or to surface related states. The dotted lines - the band structure calculated by Walter et al. [8].

energy of $5.4 \mathrm{eV}$ (3.6 eV below the valence band edge) corresponds well to the discontinuous behaviour of the $s p^{3}$ band overlapping with the flat $d$ band, due to the non-crossing rule [7].

Two features with weak dispersion, represented by open dots (at 4.7 and $6.3 \mathrm{eV}$ ) can be interpreted as related to density-of-states effects (i.e. emission from band edges at the $K$ point) or from surface related states. Both assignments would be consistent with the results of the angle-resolved photoemission study of $\mathrm{ZnTe}(110)$ by $\mathrm{Qu}$ et al. [9]. Moreover, the feature at $4.7 \mathrm{eV}$ contributes, for high detection angles, to the increased emission clearly related to the presence of $\mathrm{Mn}$ (see Fig. 2). Thus, it seems to be impossible to disentangle Mn- and ZnTe-related contributions forming this part of the observed spectra.

\section{Summary}

The cleaved and Mn doped ZnTe(110) $(1 \times 1)$ surface has been studied by angle-resolved photoemission in order to compare the band structures and to reveal changes brought about by the presence of Mn. The experimental band structure of $\mathrm{Mn} / \mathrm{ZnTe}$ along the $\Gamma-K$ direction of the Brillouin zone was derived from the set of off-normal angle-resolved photoemission spectra. The obtained $E(k)$ diagram was compared with available results of band structure calculations. The features 
related to overlap of $\mathrm{Mn} 3 d$ states with $s p^{3}$ bands - additional contribution to the density of states in the whole valence band and distortion of the band at $3.6 \mathrm{eV}$ below the valence band in the middle of the $\Gamma-K$ line - were revealed in the acquired experimental results.

\section{Acknowledgments}

The authors wish to thank Prof. A. Mycielski for supplying them with ZnTe crystals. The work was supported by MNiI (Poland) projects 1 P03B 05326 and 72/E-67/SPB/DESY/P-03/DWM68 /2004-2006 as well as the European Community — Research Infrastructure Action under the FP6 "Structuring the European Research Area" Programme.

\section{References}

[1] T. Dietl, A. Haury, Y. Merle d'Aubigné, Phys. Rev. B 55, R3347 (1997).

[2] T. Yasuda, M. Dang Duc, Y. Segawa, J. Cryst. Growth 214/215, 159 (2000).

[3] M. Ghali, J. Kossut, E. Janik, K. Regiński, Ł. Kłopotowski, P. Dłużewski, M. Potemski, F. Teran, Physica E 13, 547 (2002).

[4] A. Wall, A. Raisanen, G. Haugstad, L. Vanzetti, A. Franciosi, Phys. Rev. B 44, 8185 (1991).

[5] S. Hüfner, Photoelectron Spectroscopy. Principles and Applications, Springer-Verlag, Berlin 2003, p. 650.

[6] R.K. Swank, Phys. Rev. 153, 844 (1967).

[7] J. Mašek, B. Velický, Phys. Status Solidi B 140, 135 (1987).

[8] J.P. Walter, M.L. Cohen, Y. Petroff, M. Balkanski, Phys. Rev. B 1, 2661 (1970).

[9] H. Qu, J. Kanski, P.O. Nilsson, U.O. Karlsson, Phys. Rev. B 43, 9843 (1991). 